\title{
A UTILIZAÇÃO DO PARTOGRAMA PELA ENFERMAGEM NO TRABALHO DE PARTO SEM DISTOCIA
}

\author{
Marcia Jaqueline de LIMA ${ }^{1}$ \\ Amélia Rezende LEITE ${ }^{2}$ \\ Verusa Fernandes DUARTE ${ }^{3}$ \\ Eguimara de Souza Borges FERNANDES ${ }^{4}$ \\ Natália Teixeira FERNANDES ${ }^{5}$
}

\begin{abstract}
${ }^{1}$ Enfermeira formada pela Faculdade de Enfermagem Nova Esperança de Mossoró - FACENE/RN, docente e professora de práticas na FACENE/RN. E-mail: marciajes@facenemossoro.com.br

${ }^{2}$ Enfermeira. Formada Pela Universidade do Estado do Rio Grande do Norte - UERN. Mestre em Saúde e Sociedade pela Universidade do Estado do Rio Grande do Norte - UERN. E-mail: amelia_resende @ hotmail.com

${ }^{3}$ Verusa Fernandes Duarte. Enfermeira, Servidora Pública da Secretaria Municipal de Mossoró, Professora Especialista em Saúde Coletiva. E-mail: verusafd@facenemossoro.com.br

${ }^{4}$ Enfermeira Especialista em Enfermagem Clínica e Unidade Terapia Intensiva pela Faculdade Metropolitana de Ciências e Tecnologia FAMEC. E-mail: eguimarasouzab@ hotmail.com

${ }^{5}$ Enfermeira Especialista em Saúde da Família pela Universidade Federal de Pelotas, RS. Enfermeira da residência multiprofissional da UERN (RN). E-mail: natalia_tfernandes14@ hotmail.com
\end{abstract}

\section{Recebido em: 07/03/2016 - Aprovado em: 28/03/2017 - Disponibilizado em: 01/07/2017}

\begin{abstract}
RESUMO
A presente pesquisa teve como objetivo identificar e discutir o estado da arte sobre as publicações na utilização do partograma pela enfermagem no trabalho de parto sem distócia. Trata-se de uma pesquisa integrativa realizada através de bases de dados, observando os aspectos metodológicos e a familiaridade entre os resultados encontrados, nos permitiu elaborar as categorias: A utilização inadequada do partograma pelo enfermeiro durante trabalho de parto normal sem distorcia e a importância da utilização do partograma no trabalho de parto. Na primeira categoria mostrou que 404 partos normais foram calculados com base em uma estimativa da existência do partograma em apenas $20 \%$ dos prontuários, com precisão de $4 \%$, espaço de confiança de $95 \%$ e com perdas estimadas de $5 \%$. E na segunda constatamos que diversos estudos têm mostrado a importância do partograma tanto para o diagnóstico das alterações da evolução do trabalho de parto, como para a efetivação de intervenções em tempo hábil, garantindo o bem-estar maternofetal. O partograma se configura um excelente instrumento norteador na assistência a mulher no trabalho de parto e direcionamento a tomada de decisões para que seja feita as intervenções necessárias, mas a pesquisa mostra que apesar da sua importância é pouco utilizado ou utilizado de forma incompleta comprometendo assistência a mulher no processo de trabalho de parto.
\end{abstract}

Palavras-chave: enfermagem, partograma, parto.

\begin{abstract}
This research aimed to identify and discuss the state of the art publications on the use of the partograph by nursing in without dystocia labor. It is an integrative research conducted through databases, noting the methodological aspects and the familiarity between the results obtained allowed us to develop categories: Improper use of the partograph by nurses during normal labor without distorting and the importance of use of partograph in labor. In the first category showed that 404 normal deliveries were calculated based on an estimate of the existence of the partograph in only $20 \%$ of records, with accuracy of $4 \%$, reliable space of $95 \%$ and estimated losses of $5 \%$. And the second found that several studies have shown the importance of partogram for both the diagnosis of changes in the evolution of labor, as to the effectiveness of interventions in a timely manner, ensuring maternal and fetal well-being. The partograph is configured an excellent guiding instrument in assisting women in labor and targeting decision-making to be made the necessary interventions, but research shows that despite their importance is little used or used incompletely pledging assistance woman in labor process.
\end{abstract}

Keywords: nursing; partgram; parturition. 


\section{Introdução}

Segundo a Organização Mundial de Saúde, é considerável aceitável uma porcentagem de 10 a $15 \%$ de cesarianas e $85 \%$ para partos normais, expectativas estas que não estão de acordo com a nossa realidade. (NUNES; RAMOS; MESQUITA, 2014)

No final do século XVI, a profissão de parteira sofreu declínio, a partir do uso do fórcipe obstétrico, sua utilização influenciou a aceitação da obstetrícia como uma disciplina técnica, científica e dominada pelo homem, acreditou-se no conceito de que é possível comandar o nascimento, e que o parto era perigoso, sendo imprescindível a presença de um médico. Devido às parteiras não terem condições financeiras de adquirir o fórcipe, de serem incapazes de dominar novas técnicas por serem consideradas intelectualmente inferiores aos homens, ligado ao fato de não serem condizentes com o paradigma intervencionista médico, passaram a rejeitá-lo. (NAGAHAMA; SANTIAGO; 2005)

No Brasil, a atenção à mulher em processo parturitivo, até o século XIX, foi desenvolvida por parteiras, estando inserida no ambiente domiciliar e considerada uma prática somente feminina, na qual a parturiente recebia o apoio de outras mulheres de sua família ou de parteiras da comunidade, vivenciando este momento natural e único em suas vidas. (SANTOS; PEREIRA;

CARVALHO et al, 2012)

Santos, Pereira, Carvalho et al, 2012, relatam que com a institucionalização do parto no Brasil na década de 40 do século $\mathrm{XX}$, o profissional médico assumiu o papel hegemônico da assistência. O parto então passa a ter caráter patológico, caracterizado pela invasão na sua fisiologia, através da utilização de técnicas obstétricas, falta de privacidade para a parturiente, sendo esta submetida a tratamento de decisão médica. Este processo foi decisivo para afastar a família do nascimento.

De acordo com Nunes; Ramos; Mesquita 2014, o parto passou a ser um evento institucionalizado, programado, deixando de ser um processo natural e essencial à fisiologia feminina.

Reduzir a mortalidade materna e a infantil é algumas das metas a serem alcançadas pela Declaração do Milênio até 2015. Cerca dois milhões de recém-nascidos morrem anualmente em todo o mundo nas primeiras 24 horas de vida. Da mesma forma, aproximadamente 600.000 mulheres morrem de complicações relacionadas à gravidez, ao parto e ao puerpério. (GIGLIO; FRANÇA; LAMOUNIER, 2011)

Surgiu a partir da década de 1980 um movimento mundial em prol da humanização do parto e nascimento, uma preocupação 
crescente em dar lugar a novos paradigmas que valorizassem e atendessem o ser humano em sua integralidade, e que estimulassem os profissionais de saúde a repensarem sua prática, buscando a transformação da realidade no cotidiano do cuidado. (NAGAHAMA; SANTIAGO, 2005)

Segundo Busanello 2011, estudos e pesquisas sobre a saúde da mulher tem sido desenvolvido pela enfermagem, em defesa do PHPN e da utilização das práticas preconizadas por este programa. $\mathrm{O}$ enfermeiro tem sido reconhecido pelo MS como o profissional de saúde que possui formação holística e busca atuar de forma humanizada no cuidado à parturiente.

O partograma é um instrumento utilizado pelos profissionais de saúde que consiste na representação gráfica do trabalho de parto, e pode ser considerado um excelente recurso visual para avaliar a dilatação cervical e a descida da apresentação, em relação ao tempo. (ROCHA; OLIVEIRA; SCHNECK, et al, 2009)

\section{Objetivo}

Identificar e discutir o estado da arte sobre as publicações na utilização do partograma pela enfermagem no trabalho de parto sem distócia.

\section{Método}

Trata-se de uma pesquisa integrativa realizada através de bases de dados, cujo método é proporcionar síntese dos vários artigos publicados e explicitar a experiência vivida, permitindo assim a compreensão sobre a importância da utilização do partograma no trabalho de parto normal sem distocia acompanhado pelo enfermeiro. Artigo elaborado para obtenção de nota e titulo de especialista em obstetrícia.

A revisão integrativa consiste num método de pesquisa que nos permite construir conclusões gerais sobre uma determinada área de estudo, alcançada de forma sistemática e ordenada, com o objetivo de contribuir para o conhecimento do tema investigado que inclui a análise de pesquisas relevantes que dão base para a tomada de decisão e o melhoramento da prática clínica (VELHO, et al, 2012).

Inicialmente levantou-se a problemática, seguido das demais etapas propostas, a saber: seleção da amostragem categorização dos estudos; avaliação dos estudos (análise dos dados); discussão e apresentação dos resultados; apresentação da revisão integrativa e síntese do conhecimento. Ao fazer a busca por artigos relativos a temática identificou a carência de estudos sobre o assunto, a princípio seriam utilizados apenas artigos atuais, mas devido à escassez foram utilizados referencias mais antigas

Para a elaboração desta revisão integrativa foram transcorridas três etapas. A primeira etapa consistiu na identificação do tema e seleção da questão de pesquisa, que se 
desenvolveu a partir da temática parto normal e partograma. Assim, foi delimitada a seguinte questão de pesquisa: Qual o estado da arte sobre as publicações na utilização do partograma pela enfermagem no trabalho de parto sem distócia.

$\mathrm{Na}$ segunda etapa, foram delimitados os critérios para inclusão e exclusão dos estudos. Foram definidos como critérios de inclusão: pesquisas publicadas em forma de artigo, em periódicos nacionais e internacionais, em português que investigaram parto sem distócia o parto normal e a utilização do partograma por enfermeiros; publicadas no período de 2005 a 2015; independentemente do método de pesquisa; e que possuíam título e resumos disponíveis e indexados nas bases de dados.

A busca dos artigos foi realizada pela internet, nas bases de dados: medline, lilacs, bdenf, scielo, literaturas atuais sobre parto com foco principal em partograma. Foram selecionados os seguintes Descritores em Ciências da Saúde (DeCS), na Biblioteca Virtual de Saúde: enfermagem, parto normal, partograma.

Na terceira etapa, foi realizada uma análise crítica dos estudos selecionados, observando os aspectos metodológicos e a familiaridade entre os resultados encontrados, o que permitiu elaborar as categorias: A utilização inadequada do partograma pelo enfermeiro durante trabalho de parto normal sem distorcia e a importância da utilização do partograma no trabalho de parto.

A discussão e interpretação dos resultados, quarta etapa dessa revisão, consistiu em elaborar as recomendações para a prática, a partir das conclusões advindas da revisão, bem como, apresentar sugestão de novas pesquisas, com a identificação de lacunas nos estudos incluídos.

\section{Resultados e discussão}

A utilização do partograma pelo enfermeiro durante trabalho de parto normal sem distorcia

A utilização do partograma foi evidenciada em apenas um terço dos prontuários analisados e oito em cada dez prontuários não apresentavam anamnese completa, ou seja, nenhum dos prontuários analisados apresentou registros completos dos dados pesquisados, as informações referentes ao exame físico geral e obstétrico constavam somente em apenas três de cada dez prontuários. (BUSANELLO, 2011)

A baixa qualidade da assistência pôde ser documentada também pela mínima utilização de procedimentos, ou seja, práticas eficazes que devem ser incentivadas ressaltam-se a baixa utilização do partograma e a alta utilização de ocitocina durante o trabalho de parto, sendo que nenhum dos partos estudados foi monitorado por cardiotocografia, prática recomendada para 
acompanhamento dos partos induzidos ou conduzidos por ocitócicos. (GIGLIO;

FRANÇA; LAMOUNIER, 2011)

De acordo com estudo o número de consultas pré-natal $73,3 \%$ tinham 6 ou mais consultas e apenas 01 mulheres não haviam realizado nenhuma consulta. Em relação ao tipo de parto $66,6 \%$ era normal e 33,4 cesariana. E quanto aos registros dos mesmos pacientes mostrou que $100 \%$ dos partogramas não foram preenchidos. (SILVA; SILVA; SANTOS, et al, 2014)

Em uma amostra com 404 partos normais foi calculado com base em uma estimativa da existência do partograma em apenas $20 \%$ dos prontuários, com precisão de $4 \%$, espaço de confiança de $95 \%$ e com perdas estimadas de 5\%. (GIGLIO; FRANÇA; LAMOUNIER, 2011)

Gestantes mais velhas utilizaram menos as práticas não farmacológicas para o alívio da dor e trabalho de parto monitorado por partograma, no entanto todas as boas práticas foram mais utilizadas em primíparas, exceto o uso de partograma. (LEAL; PEREIRA; DOMINGUES, et al. 2014)

Os dados desta pesquisa contribuem para o conceito mais detalhado da utilização do partograma, instrumento que, embora recomendado pela OMS, desde 1994, vem sendo pouco utilizado nas maternidades e centros de partos intra e extra-hospitalares.
(ROCHA; OLIVEIRA; SCHNECK, et al, 2009)

A importância da utilização do partograma pelo enfermeiro no trabalho de parto sem distócia

$\mathrm{Na}$ assistência ao trabalho de parto, o uso adequado do partograma é uma prática que demanda treinamento de baixa complexidade e tem muito baixo custo, sendo assim um instrumento simples e barato, mas que pode ter um grande impacto benéfico, especialmente nessa transição de modelos de assistência obstétrica. (GATS, 2015)

Diversos estudos têm mostrado a importância do partograma tanto para o diagnóstico das alterações da evolução do trabalho de parto, como para a efetivação de intervenções em tempo hábil, garantindo o bem-estar materno-fetal. (GIGLIO; FRANÇA; LAMOUNIER, 2011)

Segundo Busanello, 2011, o partograma, representação gráfica do trabalho de parto, é um instrumento importante na prática que permite acompanhar a evolução da parturição, a dilatação cervical e a descida fetal, documentar, diagnosticar alterações, sugerir a tomada de condutas adequadas para a correção de prováveis desvios e evitar intervenções desnecessárias.

É necessária a reflexão sobre a melhoria da prática clínica destes profissionais da saúde uma nova abordagem 
que estimule a utilização do partograma como recurso clínico para a avaliação das condições do trabalho de parto e das condutas adotadas, baseando-se em evidências comprovadamente benéficas e o uso de novos métodos de cuidado que proporcionem o alívio da dor e o conforto da parturiente. (SANTOS; PEREIRA; CARVALHO et al, 2012)

Silva; Silva; Santos, et al, 2014, acreditam que embora haja algumas deficiências, como a falta de aconselhamento pré-teste / HIV; de comunicação e de preenchimento do partograma na maioria das mulheres participantes estudo, mesmo assim estão satisfeitas com o atendimento recebido na instituição de pesquisa. Entretanto, os achados sugerem que os esforços em estimular o preenchimento dessas lacunas se faz necessário para uma assistência adequada.

Para análise da qualidade do processo da assistência ao parto normal, tem sido recomendada a comparação de métodos usuais na assistência com normas e procedimentos indicados na literatura como padrão- ouro, além de alguns indicadores de processo, tais como: a utilização do partograma no prontuário e a porcentagem de partos assistidos por profissional de saúde. (GIGLIO; FRANÇA; LAMOUNIER, 2011)

Os profissionais da saúde como a enfermeira obstétrica que acompanhava, juntamente com o médico, o trabalho de parto de todas as gestantes de acordo com a evolução documentada no partograma, monitorizava o batimento cardiofetal, realizava cardiotocografias e utilizava procedimentos não farmacológicos para o alivio da dor. (VOGT, SILVA; DIAS; et al, 2014)

O partograma instrumento elaborado que representa graficamente o trabalho de parto ocorreu para $100 \%$ das mulheres, permitindo acompanhamento de sua evolução, a identificação de alterações, a tomada de condutas apropriadas para correção de desvios e prevenção de intervenções desnecessárias; o que garantiu uma adequada evolução do trabalho de parto. (VELHO; OLIVEIRA; SANTOS, 2010)

Quadro 1 - Descrição dos estudos incluídos na revisão integrativa

\begin{tabular}{|c|c|c|c|c|}
\hline Autores & Objetivo & $\begin{array}{l}\text { Instrumento para coleta } \\
\text { de dados }\end{array}$ & $\begin{array}{c}\text { Ano de } \\
\text { publicação }\end{array}$ & País \\
\hline $\begin{array}{l}\text { Barros, L. A; } \\
\text { Veríssimo, R. C. } \\
\text { S. S }\end{array}$ & $\begin{array}{l}\text { Determinar a frequência de } \\
\text { uso do partograma nas } \\
\text { maternidades escola de } \\
\text { Alagoas, Brasil. }\end{array}$ & $\begin{array}{l}\text { Foi utilizado } \\
\text { instrumento de coleta } \\
\text { de } \\
\text { dados estruturado, que foi } \\
\text { preenchido } \\
\text { pesquisador. }\end{array}$ & 2011 & Brasil \\
\hline Busanello, J. et al & Analisar & Entrevistas & 2011 & Brasil \\
\hline
\end{tabular}




\begin{tabular}{|c|c|c|c|c|}
\hline & $\begin{array}{l}\text { desenvolvidas na assistência à } \\
\text { parturiente adolescente, a } \\
\text { partir do relato dos } \\
\text { trabalhadores da saúde }\end{array}$ & $\begin{array}{l}\text { semiestruturadas, bem como } \\
\text { perguntas abertas; pesquisa } \\
\text { documental no prontuário de } \\
\text { adolescentes internadas em } \\
\text { trabalho de parto }\end{array}$ & & \\
\hline $\begin{array}{l}\text { Giglio, } \quad \text { M.R.P; } \\
\text { França, } \quad \text { E; } \\
\text { Lamounier, J.Á }\end{array}$ & $\begin{array}{l}\text { Avaliar a qualidade da } \\
\text { assistência hospitalar ao parto } \\
\text { normal em Goiânia. }\end{array}$ & $\begin{array}{lcr}\text { Entrevistas } & \text { com } & \text { as } \\
\text { puérperas e } & \text { revisão } & \text { dos } \\
\text { prontuários médicos } & \end{array}$ & 2011 & Brasil \\
\hline $\begin{array}{ll}\text { Leal, } \quad \text { M. } & \text { C; } \\
\text { Pereira, A.P. } & \text { E; } \\
\text { Domingues, } & \text { R. } \\
\text { M. S. M. et al } & \end{array}$ & $\begin{array}{l}\text { Descrever as boas práticas de } \\
\text { atenção ao parto }\end{array}$ & $\begin{array}{l}\text { Entrevistas face a face com } \\
\text { as puérperas durante a } \\
\text { internação hospitalar }\end{array}$ & 2014 & Brasil \\
\hline $\begin{array}{l}\text { Nagahama, E. E. } \\
\text { I; Santiago, S. M }\end{array}$ & $\begin{array}{l}\text { Abordar o conceito de } \\
\text { institucionalização da atenção } \\
\text { à saúde }\end{array}$ & Pesquisa descritiva. & 2005 & Brasil \\
\hline 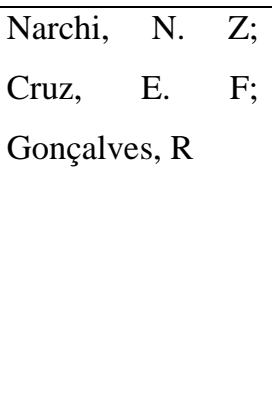 & $\begin{array}{l}\text { Oferecer subsídios teóricos } \\
\text { que sustentem a proposta de } \\
\text { que a promoção da } \\
\text { maternidade segura requer a } \\
\text { efetiva participação de } \\
\text { obstetrizes e enfermeiras } \\
\text { obstetras }\end{array}$ & Pesquisa descritiva & 2013 & Brasil \\
\hline $\begin{array}{l}\text { Rocha, I. M. S; } \\
\text { Oliveira, S. M. J. } \\
\text { V; Schneck, C. } \\
\text { A. et al }\end{array}$ & $\begin{array}{l}\text { Analisar o uso de intervenções } \\
\text { obstétricas. }\end{array}$ & $\begin{array}{l}\text { Estudo transversal, utilizado } \\
\text { o teste Qui-quadrado }\end{array}$ & 2009 & Brasil \\
\hline $\begin{array}{l}\text { Santos, } \\
\text { Pereira, } \\
\text { Carvalho, } \\
\text { E.S.C; } \\
\text { et al. }\end{array}$ & $\begin{array}{l}\text { Analisar a percepção das } \\
\text { puérperas quanto à atenção } \\
\text { recebida durante o processo } \\
\text { parturitivo em } \\
\text { maternidade pública de } \\
\text { de Seira } \\
\text { de Santana-Bahia }\end{array}$ & $\begin{array}{l}\text { Entrevistas semiestruturadas } \\
\text { com dezenove puérperas }\end{array}$ & 2012 & Brasil \\
\hline $\begin{array}{l}\text { Silva, } \\
\text { Silva, }\end{array}$ & $\begin{array}{lcr}\text { Investigar a } & \text { qualidade } & \text { da } \\
\text { assistência } & \text { oferecida } & \text { à } \\
\text { parturiente } & \text { em } & \text { uma } \\
\text { maternidade } & & \end{array}$ & Entrevistas com puérperas & 2014 & Brasil \\
\hline $\begin{array}{l}\text { Souza, M. G, } \\
\text { Vieira, } \quad \text { B.D.G; } \\
\text { Alves, V.H. et al. }\end{array}$ & $\begin{array}{l}\text { Conhecer as preocupações das } \\
\text { mulheres primíparas acerca do } \\
\text { trabalho de parto e parto; } \\
\text { Identificar ações do enfermeiro }\end{array}$ & $\begin{array}{l}\text { Pesquisa descritiva, } \\
\text { exploratória, de natureza } \\
\text { qualitativa, com seis } \\
\text { mulheres primíparas do }\end{array}$ & 2015 & Brasil \\
\hline
\end{tabular}




\begin{tabular}{|c|c|c|c|c|}
\hline & $\begin{array}{l}\text { para amenizar os sentimento } \\
\text { das mulheres. }\end{array}$ & $\begin{array}{l}\text { alojamento conjunto do } \\
\text { Hospital Universitário } \\
\text { Antônio Pedro mediante } \\
\text { entrevista semiestruturada }\end{array}$ & & \\
\hline $\begin{array}{l}\text { Velho, M. B. et } \\
\text { al. }\end{array}$ & $\begin{array}{l}\text { Identificar a contribuição das } \\
\text { pesquisas desenvolvidas, em } \\
\text { âmbito nacional } \\
\text { internacional, sobre a } \\
\text { percepção do parto normal e } \\
\text { cesáreo pelas mulheres que os } \\
\text { vivenciaram. }\end{array}$ & $\begin{array}{lrr}\text { Revisão } & \text { integrativa } & \text { onde } \\
\text { foram } & \text { percorridas } & \text { seis } \\
\text { etapas } & & \\
\end{array}$ & 2012 & Brasil \\
\hline $\begin{array}{l}\text { Velho, M. B; } \\
\text { Oliveira, M. E; } \\
\text { Santos, E. K. A. }\end{array}$ & $\begin{array}{l}\text { Identificar o estado da arte da } \\
\text { produção publicada sobre a } \\
\text { atuação da enfermeira } \\
\text { obstétrica no processo do } \\
\text { nascimento. }\end{array}$ & $\begin{array}{l}\text { Pesquisa bibliográfica } \\
\text { realizada na área da saúde }\end{array}$ & 2010 & Brasil \\
\hline $\begin{array}{l}\text { Vogt, S. E; Silva, } \\
\text { K. S; Dias, M. A. } \\
\text { B. et al. }\end{array}$ & $\begin{array}{lll}\text { Comparar os modelos } \\
\text { colaborativo e tradicional na } \\
\text { assistência ao parto e } \\
\text { nascimento }\end{array}$ & $\begin{array}{l}\text { Entrevistas e levantamento } \\
\text { de prontuários }\end{array}$ & 2014 & Brasil \\
\hline $\begin{array}{l}\text { Nunes, a. C. F; } \\
\text { Ramos, d. K. R; } \\
\text { Mesquita, s. K. C. }\end{array}$ & $\begin{array}{l}\text { Preferência por cesarianas em } \\
\text { gestantes nulíparas de um } \\
\text { consultório particular de } \\
\text { ginecologia e obstetrícia: um } \\
\text { estudo de caso. }\end{array}$ & $\begin{array}{l}\text { Estudo de caso com } \\
\text { abordagem } \quad \text { qualitativa } \\
\text { enfoque } \\
\text { descritivo }\end{array}$ & 2014 & Brasil \\
\hline
\end{tabular}

Fonte: base de dados

partograma e a alta utilização de ocitocina

\section{Considerações finais}

O partograma se configura um excelente instrumento norteador na assistência a mulher no trabalho de parto e no direcionamento a tomada de decisões para que seja feita as intervenções necessárias, mas a pesquisa mostra que apesar da sua importância ele é pouco utilizado ou utilizado de forma incompleta comprometendo assistência a mulher no processo de trabalho de parto, evidenciamos a baixa utilização do durante o trabalho de parto, ou seja, impedindo a evolução espontânea do trabalho de parto, prática essa que não é recomendada, mas que é bastante utilizada pelos profissionais médicos, a uma escassez de estudos que evidencie o porquê dos profissionais da enfermagem não utilizarem ou utilizarem de forma inadequada os partogramas, sugerimos estudos sobre o tema para que seja feito um levantamento de quais dificuldades encontradas para o preenchimento correto do partograma. O uso 
adequado do partograma reduz a taxa de intervenções e cirurgias desnecessárias, facilita o processo natural do parto e com isso proporciona autonomia da mulher $\mathrm{e}$ participação ativa.

\section{Referências}

BARROS, L. A; VERÍSSIMO, R. C. S. S. Uso do partograma em maternidades escola de alagoas. Rev Rene, Fortaleza, jul/set; v3, n.12, p.555-60, 2011. Disponível:

http://www.revistarene.ufc.br/vol12n3_pdf/a1 5v12n3.pdf acesso: 18 out. 2015

BUSANELLO, J. et al. Atenção humanizada ao parto de adolescentes: análise das práticas desenvolvidas em um Centro Obstétrico. Rev Bras Enferm, Brasília 2011 set-out; v. 5, n. 64, p. 824-32. Disponível:

http://www.scielo.br/pdf/reben/v64n5/a04v64 n5.pdf acesso: 25 out. 2015

GATS (Grupo de Avaliação de Tecnologias em Saúde). Revisando o uso do partograma e seu papel nas indicações de cesariana intraparto. Centro de inovações. Belo Horizonte, 2015.

GIGLIO, M.R.P; FRANÇA, E; LAMOUNIER, J.Á. Avaliação da qualidade da assistência ao parto normal. Rev Bras

Ginecol Obstet. 2011; v. 10, n. 33, p.297-

304. Disponível:

http://www.scielo.br/pdf/rbgo/v33n10/05.pdf acesso: 03 nov. 2015

LEAL, M. C; PEREIRA, A.P. E; DOMINGUES, R. M. S. M. et al. Intervenções obstétricas durante o trabalho de parto e parto em mulheres brasileiras de risco habitual. Cad. Saúde Pública, Rio de Janeiro, 30 Sup:S17-S47, 2014. Disponível: http://www.scielo.br/pdf/csp/v30s1/0102311X-csp-30-s1-0017.pdf acesso: 30 nov. 2015

NAGAHAMA, E. E. I; SANTIAGO, S. M. A institucionalização médica do parto no Brasil. Ciênc. saúde coletiva [online]. 2005, v.3, n.10, p. 651-657. ISSN 1678-4561.
Disponível:

http://www.scielo.br/pdf/csc/v10n3/a21v10n3 .pdf acesso: 29 nov. 2015

NARCHI, N. Z; CRUZ, E. F; GONÇALVES, R. O papel das obstetrizes e enfermeiras obstetras na promoção da maternidade segura no Brasil. Ciênc. saúde coletiva v.18 n.4, Rio de Janeiro abr. 2013. Disponível:

http://www.scielosp.org/pdf/csc/v18n4/19.pdf acesso: 04 de setembro 15

NUNES, A. C. F; RAMOS, D. K. R; MESQUITA, S. K. C. Preferência por cesarianas em gestantes nulíparas de um consultório particular de ginecologia e obstetrícia: um estudo de caso. Revista da Universidade Vale do Rio Verde, Três Corações, v. 12, n. 2, p. 743-753, ago./dez. 2014. Disponível em:

http://revistas.unincor.br/index.php/revistauni ncor/article/view/1484/pdf_252

Acesso: 04 mar. 2016

ROCHA, I. M. S; OLIVEIRA, S. M. J. V; SCHNECK, C. A. et al. O partograma como instrumento de análise de assistência ao parto.

Rev. Esc. Enf. USP; v.3, n. 43, p. 880-8, 2009. Disponível:

http://www.scielo.br/pdf/reeusp/v43n4/a20v4 3n4.pdf acesso: $01 \mathrm{dez} .2015$

SANTOS, L.M, PEREIRA, S.S. C; CARVALHO, E.S.S. et al. Atenção no processo parturitivo sob o olhar da puérpera.

R. pesq.: cuid. fundam. [Online] 2012. jul./set. v3, n.4, p.2655-66. Disponível: http://www.seer.unirio.br/index.php/cuidadof undamental/article/view/1830/pdf 602 acesso: 20 ago. 2015

SILVA, F.F.A; SILVA, R.A.R; SANTOS, F.A.P.S. et al. Atendimento prestado a parturiente em um hospital universitário. J. res.: fundam. care. online 2014. jan./mar. n.61, p.282-292. Disponível:

http://www.seer.unirio.br/index.php/cuidadof undamental/article/viewFile/3002/pdf_1062 acesso: 07 nov. 2015

SOUZA, M. G, VIEIRA, B.D.G; ALVES, V.H. et al. Preocupação das mulheres primíparas em relação ao trabalho de parto e 
parto. J. res.: fundam. Care. Online, jan.mar. v1, n.7, p. 1987-2000, 2015. Disponível: http://saudepublica.bvs.br/pesquisa/resource/p t/bde-26709 acesso: 21 out. 2015

VELHO, M. B. et al. Vivência do parto normal ou cesáreo: revisão integrativa Sobre a percepção de mulheres. Texto Contexto Enf., Florianópolis, abr-jun; v. 2, n. 21, p. 458-66, 2012. Disponível em:

http://www.scielo.br/pdf/tce/v21n2/a26v21n2. pdf Acesso: 28 ago. 2015

VELHO, M. B; OLIVEIRA, M. E; SANTOS, E. K. A. Reflexões sobre a assistência de enfermagem prestada à parturiente. Rev Bras Enferm, Brasília, jul-ago; v.4, n.63, p.652-9, 2010. Disponível:

http://www.scielo.br/pdf/reben/v63n4/23.pdf acesso: $04 \mathrm{dez} 2015$

VOGT, S. E; SILVA, K. S; DIAS, M. A. B. et al. Comparação de modelos de assistência ao parto em hospitais públicos. Rev Saúde Pública; v.2, n. 48, p.304-313, 2014. Disponível: http://www.revistas.usp.br/rsp/article/view/81 154 acesso: 30 nov.2015 